\title{
Good Outcome after More Than a Million Troponin in Serum
}

\author{
Mohamed Elbayomi* ${ }^{\circledR}$, Timo Seitz, Michael Weyand \\ Department of Cardiac Surgery, Friedrich-Alexander-University Erlangen-Nuremberg, Erlangen, Germany \\ Email: *elbayomimohamed3@gmail.com
}

How to cite this paper: Elbayomi, M., Seitz, T. and Weyand, M. (2022) Good Outcome after More Than a Million Troponin in Serum. Case Reports in Clinical Medicine, 11, 25-30. https://doi.org/10.4236/crcm.2022.111005

Received: January 1, 2022

Accepted: January 18, 2022

Published: January 21, 2022

Copyright (c) 2022 by author(s) and Scientific Research Publishing Inc. This work is licensed under the Creative Commons Attribution International License (CC BY 4.0).

http://creativecommons.org/licenses/by/4.0/

(c) (i) Open Access

\begin{abstract}
A troponin in serum of more than one Million in vivo is rare insult. We report a case of 85-year-old woman who was transferred to our institution because of acute ST elevated myocardial infarction and the troponin level reached a peak of $1,032,904.2 \mathrm{pg} / \mathrm{ml}$. She was discharged with highly reduced left ventricular function, however in the three months follow up after a coronary bypass operation she completely was free from heart failure symptoms and echocardiography showed a totally normal function of both ventricles. This case study highlights that the initial serum troponin in acute settings can mislead the long-term prognosis and gives prominence to the expeditious myocardial revascularization.
\end{abstract}

\section{Keywords}

Cardiac Troponin, Coronary Bypass Operation, Heart Lung Machine

\section{Background}

Troponin plays a defining role in the diagnosis of myocardial ischemia, here we present a case of 85-year-old female patient with acute transmural myocardial infarction with diffuse ST-segment elevation in which the peak of serum troponin was more than a million $(1,032,904.2 \mathrm{pg} / \mathrm{ml})$. In the three months follow-up transthoracal echocardiography shows normal pump function and freedom of symptoms and signs of heart failure after prompt revascularization through CABG procedure.

\section{Case Presentation}

A previously physically fit 85 -year-old female presented to emergency department (ED) of a secondary referral centre with acute central chest pain. The past medical history was only pertinent for hypertension, hyperlipidaemia. Coronary 
angiography revealed completely occluded ostial LAD with triple vessel disease (Figure 1). High sensitivity Troponin upon the admission in our institution was $34,745.5 \mathrm{pg} / \mathrm{ml}$. Afterwards she was directly transferred to our operation theatre for an emergency CABG procedure. After induction of general anaesthesia, the routine perioperative transoesophageal echocardiography (TOE) supported the diagnosis showing total anterior wall and septum akinesia.

An intra-aortic balloon pump was inserted intraoperatively through the femoral artery to support the circulation. A full median sternotomy was performed, and the heart was accessed after complete opening of the pericardial sac. Cardiopulmonary bypass with Hyperkalemic normothermic blood cardioplegic arrest was utilized.

A Standard CABG operation with Internal mammary artery on the culprit coronary vessel Left anterior Descending (LAD) as an in-situ graft and 2 singel vein grafts on Obtuse marginal artery marginalis and right coronary artery was performed. The CPB time was $78 \mathrm{~min}$., and the Cross-clamp time was $42 \mathrm{~min}$. The patient was normothermic $36.8^{\circ} \mathrm{C}$. Weaning from extra-corporeal circulation was uncomplicated, after the procedure the patient was shifted to the operative intensive care unit (ICU), where she was extubated a couple of hours later. The follow up serum troponin in post operative period was more than million $(1,032,904.2 \mathrm{pg} / \mathrm{ml}$ ) (Figure 2) and CK-MB was $5134 \mathrm{U} / \mathrm{l}$. She was then transferred to the surgical ward for further post-operative care which was uneventful.

The post-operative transthoracic echocardiogram showed reduced Left ventricular ejection fraction (HFrEF) of $20 \%$ by Simpson's biplane method. Further evaluation using a cardiac MRI showed a LVEF of 10\% - 15\% (Figure 3 and Figure 4). She had complaint of progressive dyspnoea (New York Heart Association (NYHA) functional Class III).

Because of the reduced ejection fraction, the patient discharged with Wearable Cardioverter Defibrillator to minimize the high risk of sudden cardiac death and before discharge a follow-up appointment was scheduled in our outpatient clinic to re-evaluate the cardiac function after 3 months.

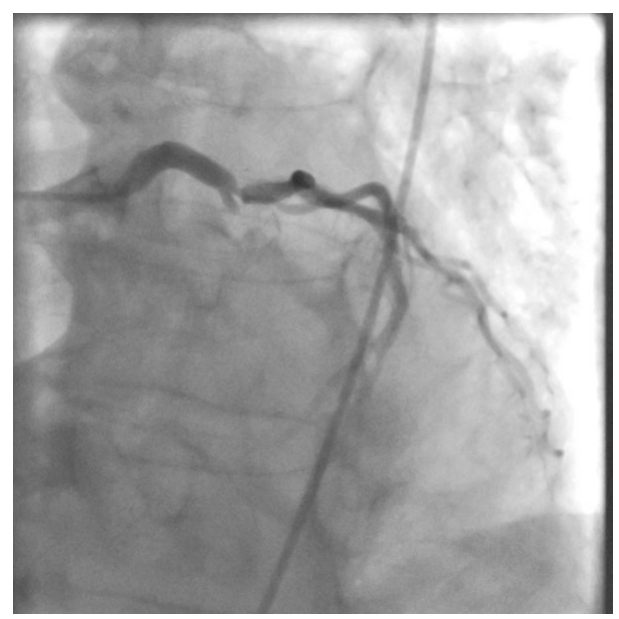

Figure 1. Coronary angiography LAO: 30 CRA: 21 shows total occlusion of LAD. 


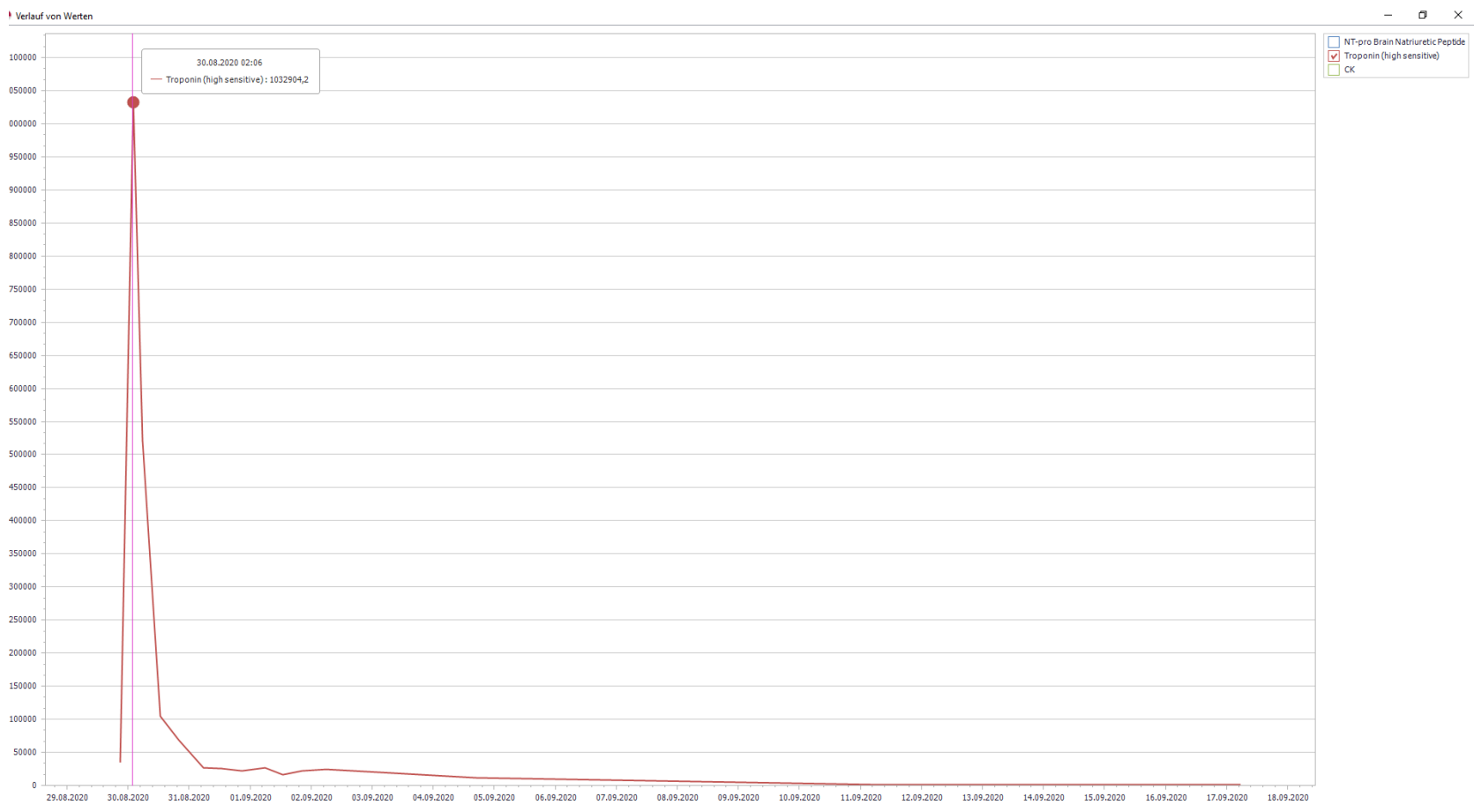

Figure 2. Peak serum level of high sensitivity troponin of 1,032,904.2 Pg/ml.

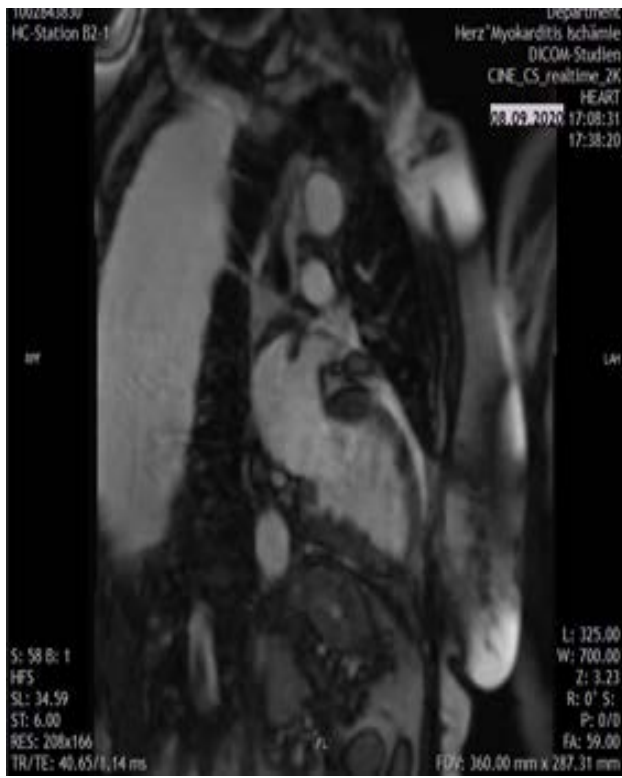

Figure 3. Cardiac magnetic resonance imaging "2 Chamber view”.

At a 3-month follow-up, she had returned to normal physical activity and was free from heart failure signs and symptoms. She underwent a transthoracic echocardiogram which showed a Left ventricle ejection fraction of $50 \%$ by Simpson's biplane method with no wall motion abnormalities (Figure 5).

\section{Discussion}

Acute myocardial infarction is a major health problem which leads to higher 


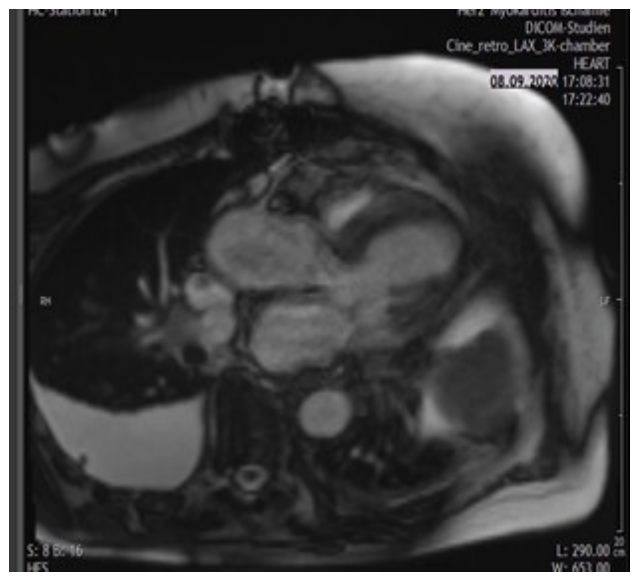

Figure 4. Cardiac magnetic resonance imaging " 3 chamber view".

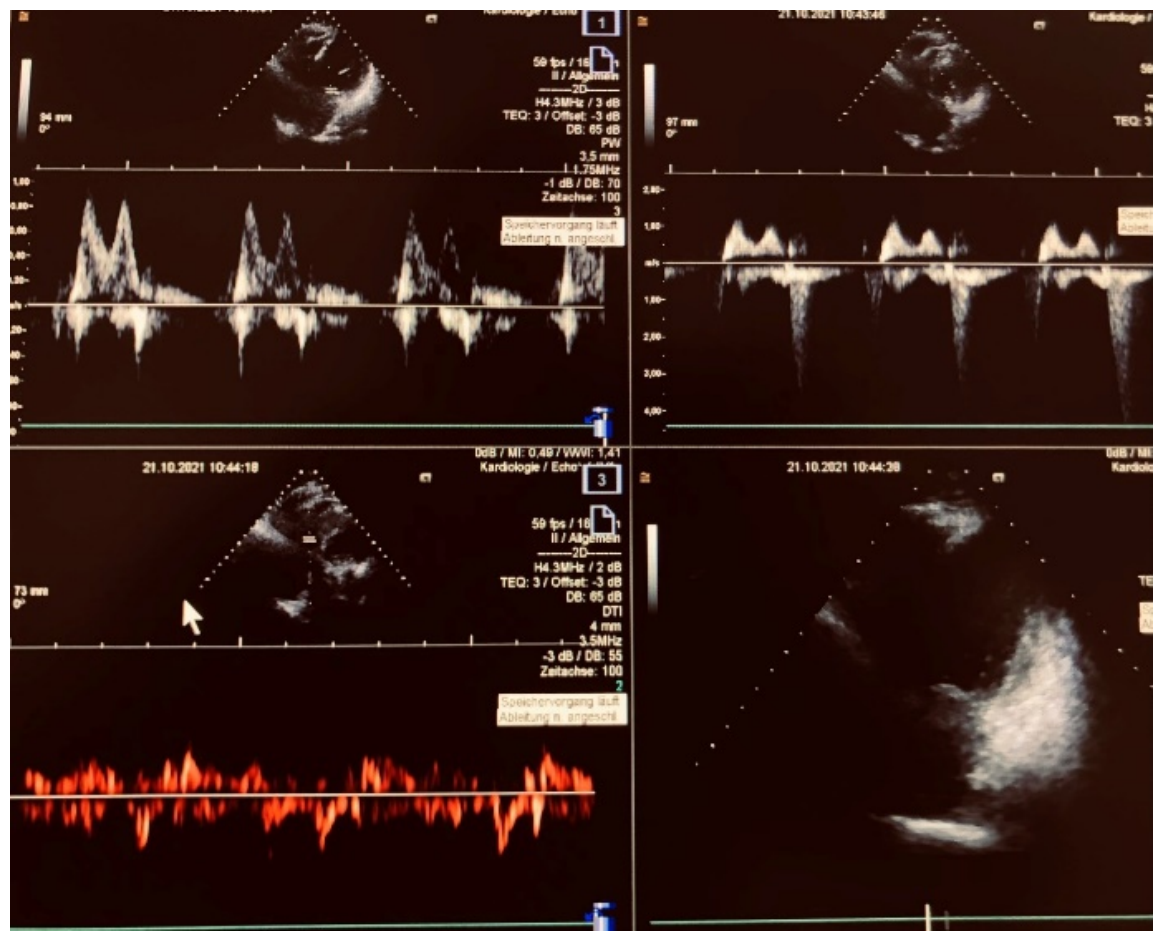

Figure 5. Transthoracic echocardiography showed a normal systolic function and normal end-diastolic filling pressures of left ventricle.

rates of mortality and morbidity. That fatal impact triggers the need of appropriate biomarkers for early diagnosis. Currently, the most specific markers for cardiac injury are cardiac troponin I (cTnI) and cardiac troponin $\mathrm{T}(\mathrm{cTn} T)$ which have been considered as "gold standard" to identify cardiac ischemia due to its high specificity. In the daily clinical practice cardiac troponins becomes a predominant indicator for MI. However, troponin leakage in serum may originate from a cytosolic compartment of the cells and not from the necrosis of thin filament [1]. The Preoperative troponin levels do not appear to be predictive of short- or long-term outcomes following CABG and the clinical decisions regarding surgical revascularization should not be dictated by these measurements 
[2]. In a study to explore the association between biomarker elevation (CK-MB and $\mathrm{cTn}$ ), following percutaneous coronary intervention (PCI), there was an increased mortality rate with elevation of CK-MB after PCI, whereas cTn elevation was not independently associated with mortality at 1 year [3].

It was concluded in an animal study that cTnI is useful in assessing the prognosis and severity of cardiac diseases in dogs as well as predicting the response to treatment can be assessed by repeated sampling, persistent increases in cTnI concentrations are indicators of a poor prognosis in dogs with cardiac disease [4].

In an experimental work it was shown that revascularization of hibernating myocardium stimulated myocyte proliferation and partially reverse the ischemia [5].

\section{Conclusion}

Serum Cardiac troponin alone is not always reliable as a prognostic factor for post-operatively morbidity and mortality, rapid revascularization can prevent a devastating consequence.

\section{Data Availability Statement}

The raw data supporting the conclusions of this article will be made available by the authors, without undue reservation to any qualified researcher.

\section{Ethics Statement}

Patient signed informed consent related to clinical course, therefore and due to its retrospective nature of the educational case report, the Institutional Review Board was waived.

\section{Author Contributions}

All authors contributed to this patient care, diagnosis, and treatment and in writing this article.

\section{Acknowledgements}

The authors would like to acknowledge all who contributed to this case diagnosis, therapy, and decision-making. Acknowledgment is given to Erlangen university hospital especially for the heart surgery and anesthesia department.

\section{Conflicts of Interest}

The authors declare that the research was conducted in the absence of any commercial or financial relationships that could be construed as a potential conflict of interest.

\section{References}

[1] Agewall, S. and Giannitsis, E. (2014) Troponin Elevation in Coronary Ischemia and 
Necrosis. Current Atherosclerosis Reports, 16, Article No. 396.

https://doi.org/10.1007/s11883-013-0396-7

[2] Hess, N.R., Sultan, I., Wang, Y., Thoma, F.W. and Kilic, A. (2021) Preoperative Troponin Levels and Outcomes of Coronary Surgery Following Myocardial Infarction. Journal of Cardiac Surgery, 36, 2429-2439. https://doi.org/10.1111/jocs.15557

[3] Garcia-Garcia, H.M., McFadden, E.P., von Birgelen, C., Rademaker-Havinga, T., Spitzer, E., Kleiman, N.S., Cohen, D.J., Kennedy, K.F., Camenzind, E., Mauri, L., Steg, P.G., Wijns, W., Silber, S., van Es, G.-A., Serruys, P.W., Windecker, S., Cutlip, D. and Vranckx, P. (2019) Impact of Periprocedural Myocardial Biomarker Elevation on Mortality Following Elective Percutaneous Coronary Intervention. JACC: Cardiovascular Interventions, 12, 1954-1962.

https://doi.org/10.1016/j.jcin.2019.07.014

[4] Fonfara, S., Loureiro, J., Swift, S., James, R., Cripps, P. and Dukes-McEwan, J. (2010) Cardiac Troponin I as a Marker for Severity and Prognosis of Cardiac Disease in Dogs. The Veterinary Journal, 184, 334-339.

https://doi.org/10.1016/j.tvjl.2009.04.004

[5] Page, B.J., Banas, M.D., Suzuki, G., Weil, B.R., Young, R.F., Fallavollita, J.A., Palka, B.A. and Canty, J.M. (2015) Revascularization of Chronic Hibernating Myocardium Stimulates Myocyte Proliferation and Partially Reverses Chronic Adaptations to Ischemia. Journal of the American College of Cardiology, 65, 684-697.

https://doi.org/10.1016/j.jacc.2014.11.040

\section{Abbreviations and Acronym}

- $\mathrm{Pg} / \mathrm{ml}$ pikogramm pro $\mathrm{ml}$

- U/l Unites pro Litre

- $\mathrm{Ck}$ Creatin-Kinase

- WHO World health Organization

- cTnI Cardiac troponin I

- $c \operatorname{TnT}$ Cardiac troponin T

- MI Myocardial infarct

- CABG Coronary Artery Bypass Grafting

- CK-MB Creatine Kinase-myocardial band

- cTn Cardiac Troponin

- PCI Percutaneous coronary intervention

- IRB Institutional Review Boards

- IABP Intra Aortal Balloon Pump 